\section{Additions to the flora of Marathwada region of Maharashtra, India}

\section{S.P. Gaikwad ${ }^{1}$, R.D. Gore ${ }^{2} \&$ K.U. Garad ${ }^{3}$ \\ 1,2,3 Life Science Research laboratory, Walchand College of Arts and Science, Solapur, Maharashtra 413006, India Email: 1sayajiraog@gmail.com, ${ }^{2}$ ramdgore@gmail.com (corresponding author), ${ }^{3}$ garadku@gmail.com}

Marathwada region comprising seven districts $\left(70^{0} 5^{\prime}-78^{0} 5^{\prime} \mathrm{N} \& 17^{0} 5^{\prime}-20^{0} 5^{\prime} \mathrm{E}\right)$ forms a part of the vast Deccan Plateau of Maharashtra, India. The plant wealth of the Marathwada region is known through publications of several researchers (Naik 1966, 1967, 1969, 1970, 1979, 1998; Lakshminarasimhan 1996; Almeida 1998, 2001, 2003, 2009; Singh \& Karthikeyan 2000, 2001). Cooke (1958 a,b,c reprint edition) in his 'Flora of Bombay Presidency' had not included Marathwada region, as it was then under Hyderabad State.

Ramling Wildlife Sanctuary and the adjoining region comprises hills and hillocks which support rich tropical dry deciduous and scrub vegetation. During our floristic explorations, thirteen taxa of flowering plants were recorded which are new to the Marathwada region.

Date of publication (online): 26 April 2012

Date of publication (print): 26 April 2012

ISSN 0974-7907 (online) | 0974-7893 (print)

Editor: Arun K. Pandey

Manuscript details:

Ms \# 02835

Received 14 June 2011

Final received 03 February 2012

Finally accepted 18 February 2012

Citation: Gaikwad, S.P., R.D. Gore \& K.U. Garad (2012). Additions to the flora of Marathwada region of Maharashtra, India. Journal of Threatened Taxa 4(4): 2515-2523.

Copyright: (C) S.P. Gaikwad, R.D. Gore \& K.U. Garad 2012. Creative Commons Attribution 3.0 Unported License. JoTT allows unrestricted use of this article in any medium for non-profit purposes, reproduction and distribution by providing adequate credit to the authors and the source of publication.

Acknowledgement: Authors are grateful to the Principal, Walchand College of Arts \& Science, Solapur for providing available research facilities; Director Botanical Survey of India, Western Circle, Pune, and Curator, Blatter Herbarium, Mumbai for confirmation of identifications, and to RGSTC Government of Maharashtra for financial assistance.

\section{OPEN ACCESS | FREE DOWNLOAD}

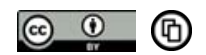

\section{Enumeration}

Acacia planifrons Wight \& Arn.

Prodr. 276. 1834. (Mimosaceae)

(Images $1 \& 2$ )

Specimen examined: Blatter 17176 (Blatt. Herb.); 17.v.2011, RDG-488.

Small armed tree with spreading branches. Leaves 2-pinnate. Stipular spines of two kinds. Flowers in globose heads in axillary fascicles. Pods sub-cylindric, turgid, circinate.

Flowering and Fruiting: October-December.

Distribution: Apsinga Road (1802'29.64”N \& 7603'17.70'E) in Osmanabad District.

Note: The species is readily distinguished from others in having an umbrella like canopy, stipular spines of two kinds and circinate pods.

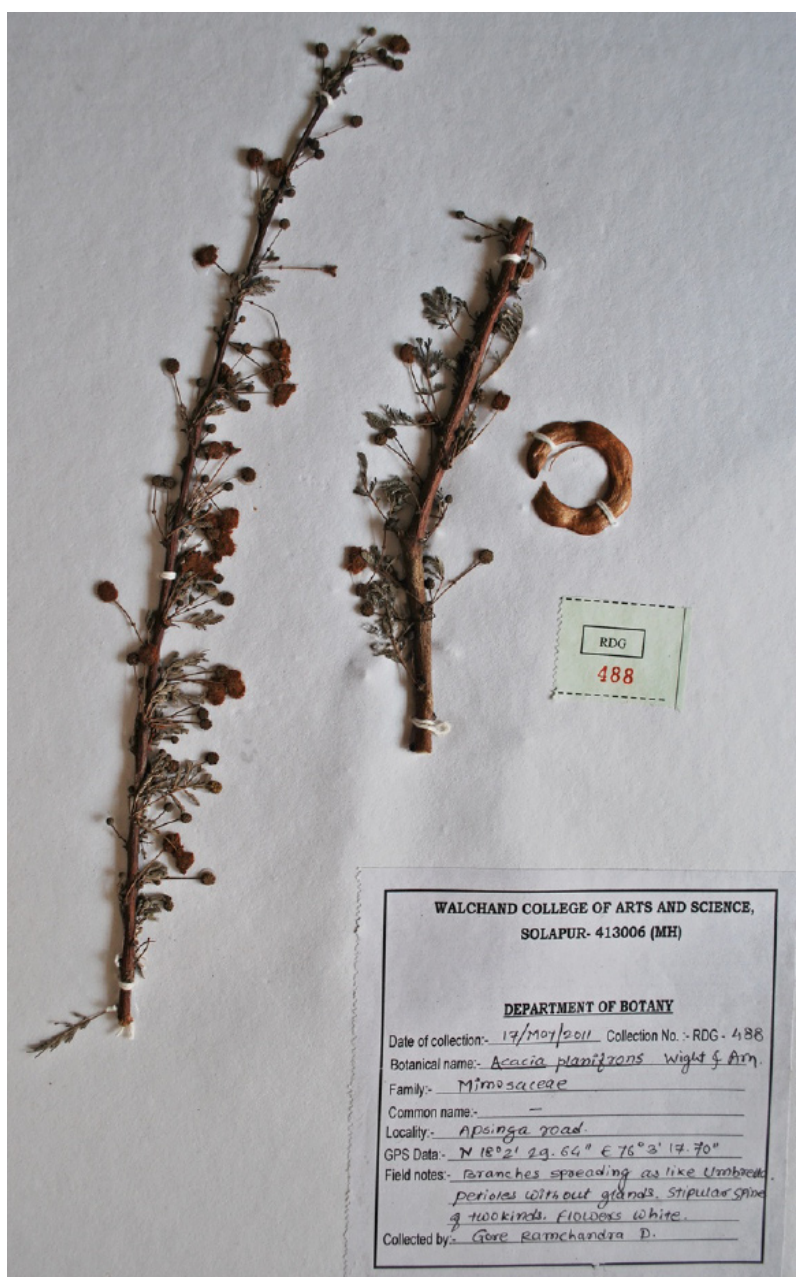

Image 1. Acacia planifrons 


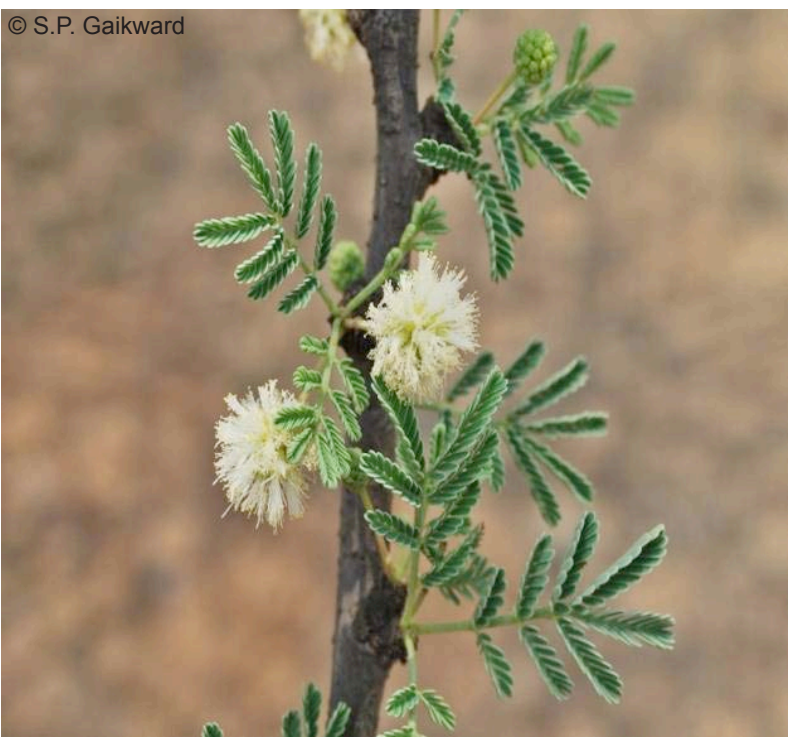

Image 2. Acacia planifrons Wight. \& Arn.

Acacia senegal (L.) Willd. Sp. Pl. 4: 1030. 1806.

Mimosa senegal L. Sp. Pl. 521. 1753. (Mimosaceae)

(Images $3 \& 4$ )

Specimen examined: Solapur R. Manakandam 126 (BNHS); 08.v.2011, RDG-491.

A small tree; stem prickly. Leaves 2-pinnate. Stipular spines usually ternate. Flowers white in spikes. Pods linear-oblong, flat, 5-6 seeded.

Flowering and Fruiting: November-April.

Distribution: Ramling Wildlife Sanctuary $\left(18^{0} 18^{\prime} 25.22^{\prime \prime} \mathrm{N} \& 7^{0} 55^{\prime} 19.92^{\prime \prime} \mathrm{E}\right)$ in Osmanabad

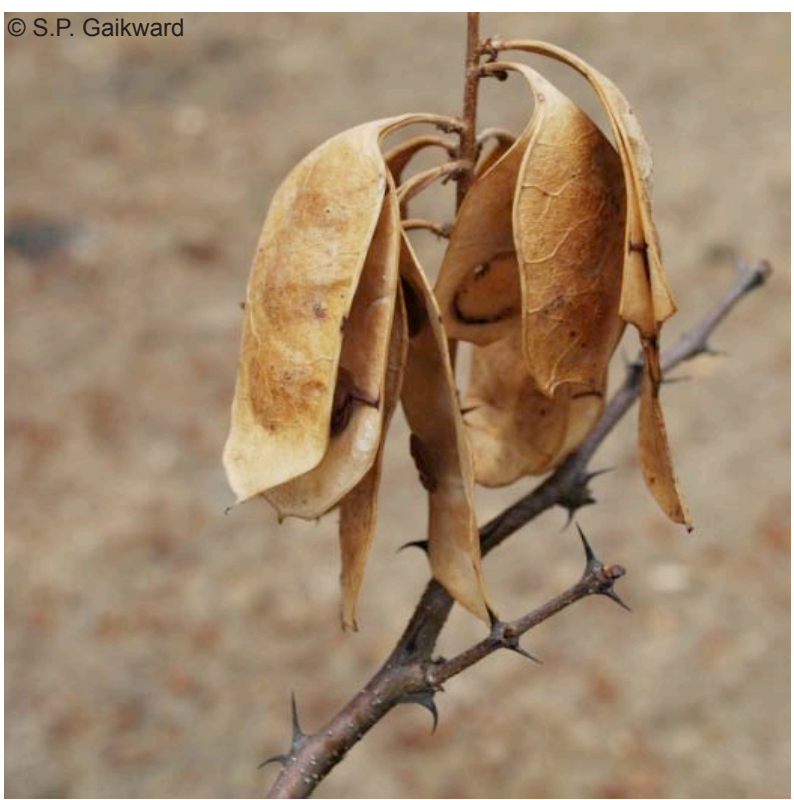

Image 3. Acacia senegal (L.) Willd.

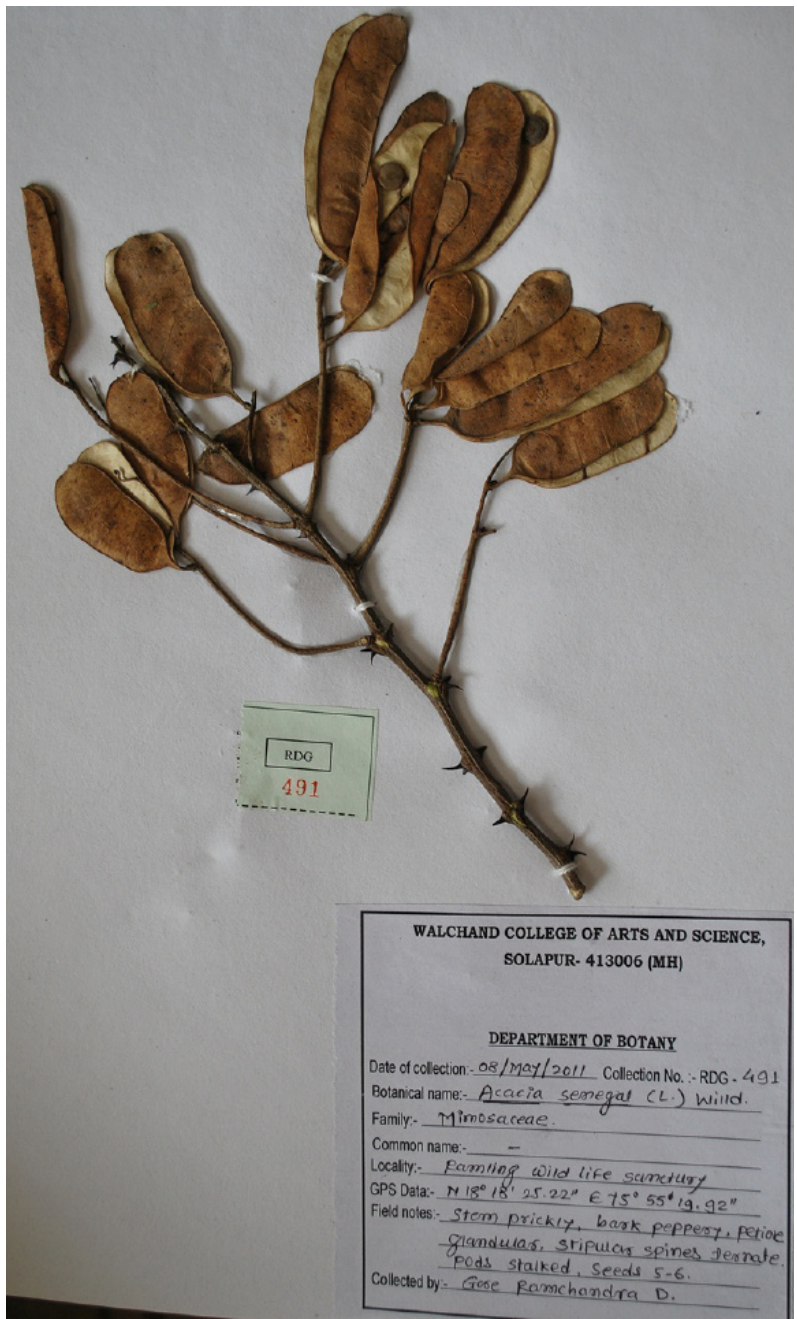

Image 4. Acacia senegal

District.

Notes: The species can be easily identified by its ternate stipular spines - the two lateral spines are nearly straight or slightly curved upwards and the middle one is curved downwards.

Aerva javanica (Burm. f.) Juss. ex. Schult. Syst. Veg. edt. 15. 5: 565.1819. Iresine javanica Burm. f. Fl. India. 217, t. 65 , f. 1. 1768. (Amaranthaceae)

(Images 5 \& 6)

Specimen examined: 02.01.2011, RDG-431.

Annual, erect, pubescent herbs. Leaves sessile, alternate, $2-5 \times 0.5-1.5 \mathrm{~cm}$. Flowers in axillary or terminal spikes, dioecious. Urticles orbicular-ovoid. Seeds lenticular, black.

Flowering and Fruiting: November-January.

Distribution: Katri (18³'39.88”N \& 7600'56.76”E) in Osmanabad District. 


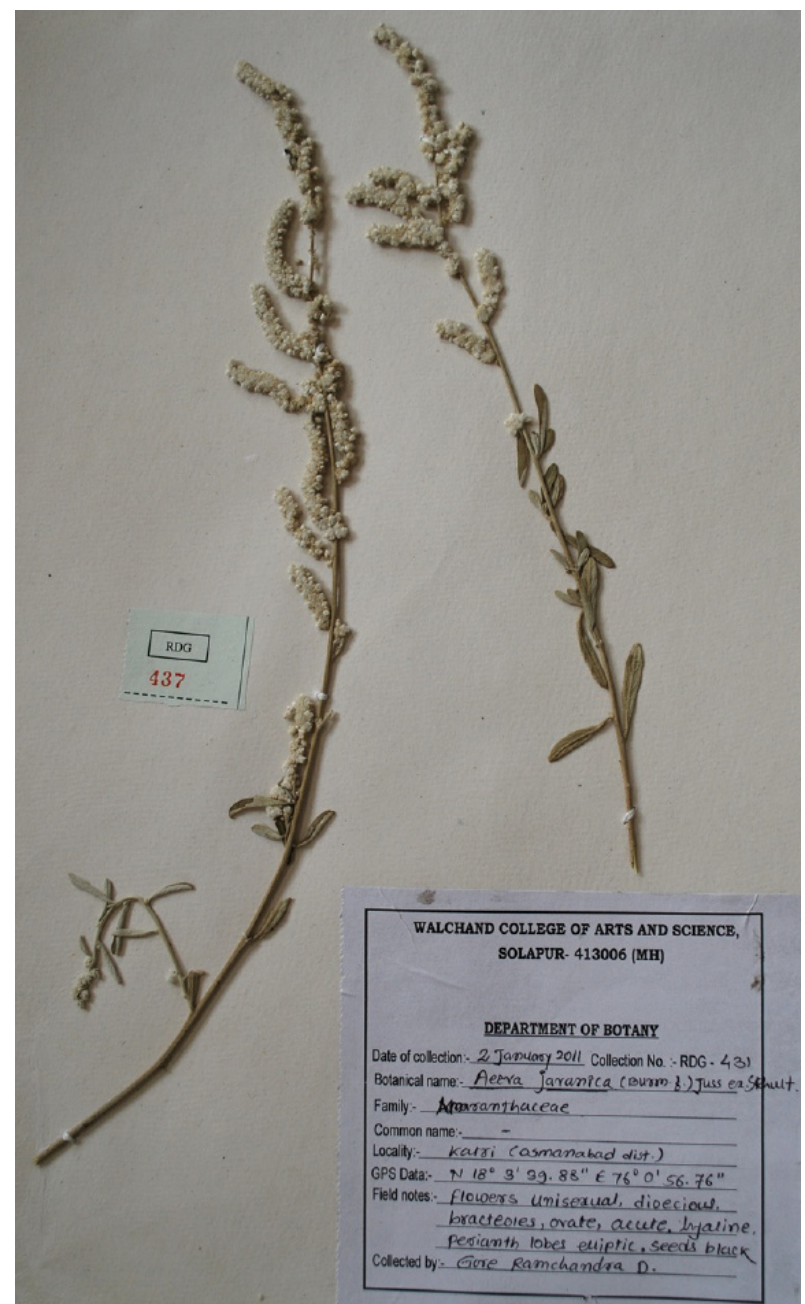

Image 5. Aerva javanica

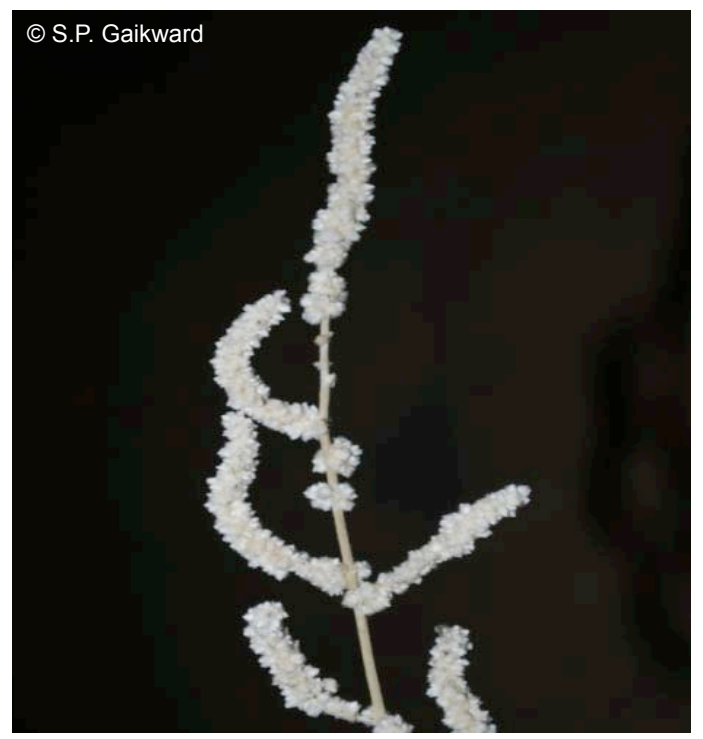

Image 6. Aerva javanica (Burm. f.) Juss. ex. Schult.

\section{Ammannia nagpurensis Matthew \& Nayar in}

Bull. Surv. India 31: 158, f.1 a-d. (1989) 1992.

(Lythraceae) (Images 7 \& 8)

Specimen examined: Starky point (Nagpur), Mirashi 252, (Blat.); 21.xi.2010, RDG-280.

Annual herbs. Leaves opposite, decussate, linearoblong. Flowers axillary in pedunculate cymes. Calyx campanulate; petals four, caducous. Stamens four. Capsules globose.

Flowering and Fruiting: October-November

Distribution: Malumbra $\quad\left(17^{0} 56{ }^{\prime} 28.62\right.$ 'N \& $76^{0} 1^{\prime} 48.72$ 'E) in Osmanabad District.

Note: So far this species was known from only the Nagpur region of Maharashtra, so the present collection has extended the range of distribution of the species.

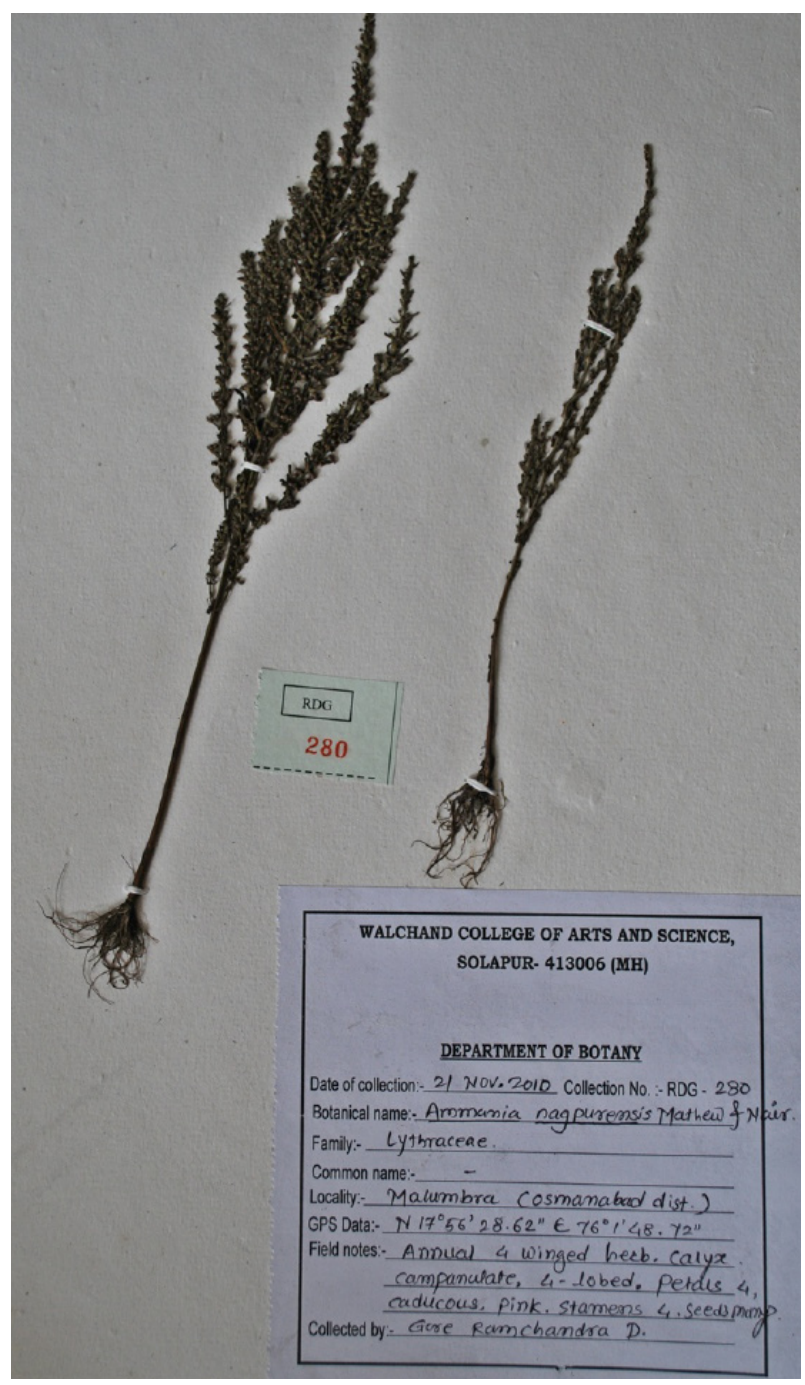

Image 7. Ammania nagpurensis 


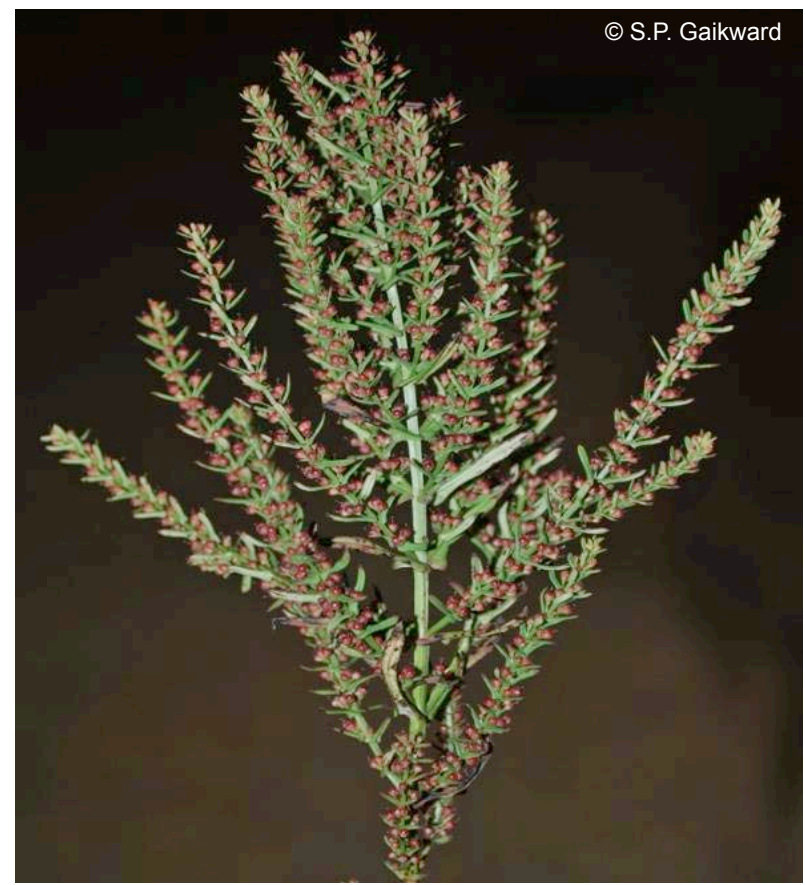

Image 8. Ammannia nagpurensis Matthew \& Nayar

Cuscuta campestris Yuncker in Mem. Torr. Bot. Club. 18: 138, f. 14. 1932. (Cuscutaceae)

(Images $9 \& 10$ )

Specimen examined: 12.ix.1960, Near Patanwadi Bridge, Pune, Wadhwa 64209; 12.xi.2010, Koregaon Park, Pune, N.P. Singh 112858 (BSI Pune); RDG351.

Stem slender, yellowish. Flowers greenish-yellow. Corolla lobes, acute; scales ovate, triangular. Stamens slender, infrastaminal scales present. Styles two; stigma globose.

Flowering and Fruiting: November-February.

Distribution: Apsinga (1804'5.29'N \& 7602'7.63”E) and Bembli $\left(18^{\circ} 9^{\prime} 18.85^{\prime} \mathrm{N}\right.$ \& $76^{\circ} 8^{\prime} 27.34$ ”'E) in Osmanabad District.

Note: Occasionally parasite on Achyranthus aspera L., Lantana camara L. \& Vitex negundo L. etc.

Dyssodia tenuifolia Loes. in Bull. Herb. Boiss. 2, 6: 866.1906. (Asteraceae) (Images $11 \& 12$ )

Specimen examined: 11.v.2011, RDG-507.

Annual herbs. Leaves sessile, deeply pinnatisect. Heads radiate, pedunculate. Involucral bracts ciliate; phyllaries persistent, each bearing 1-7 round to elliptic oil-glands. Corollas yellow. Achenes black.

Flowering and Fruiting: December-May.

Distribution: Osmanabad Town (18010`41.90”N \&

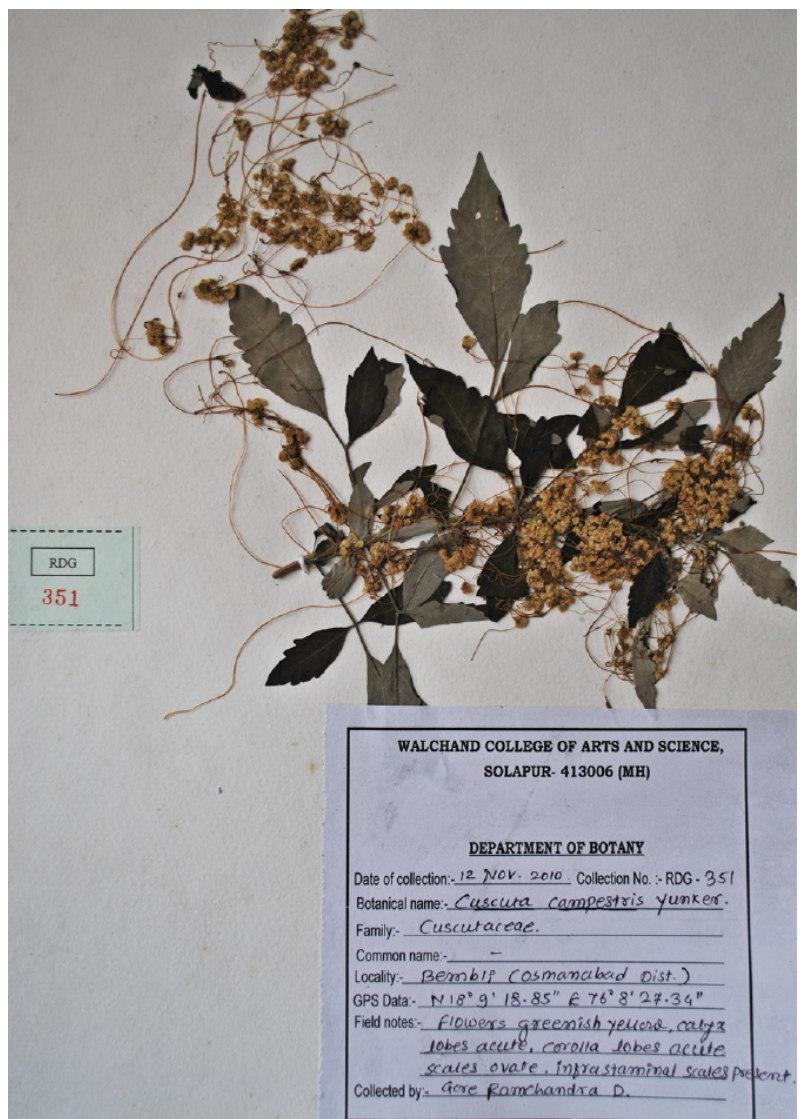

Image 9. Cuscutta campestris

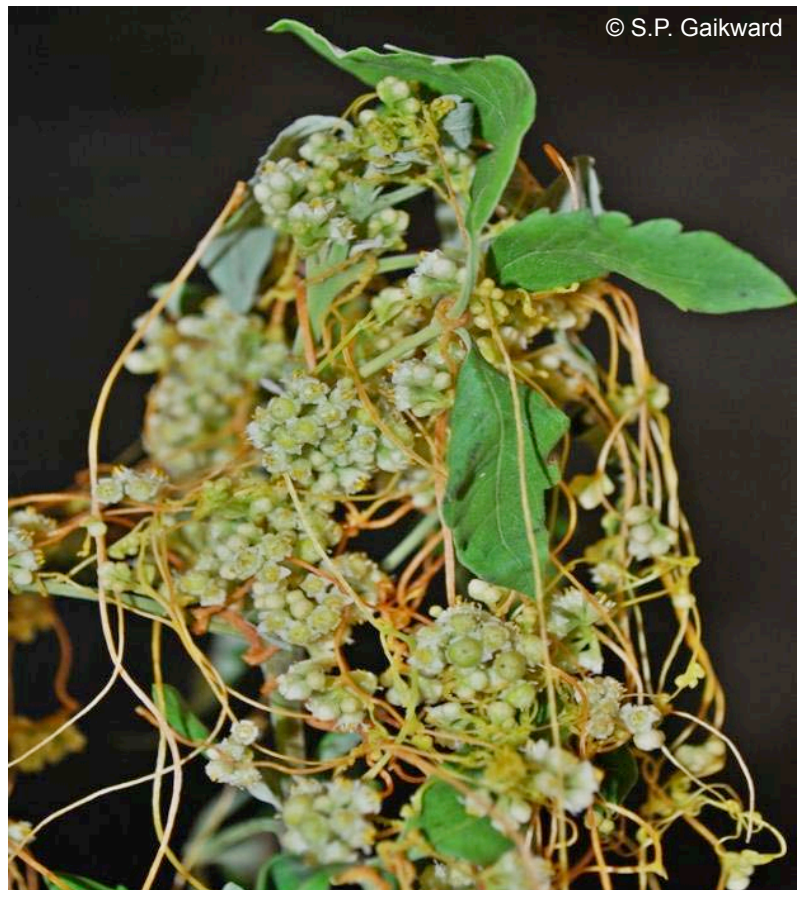

Image 10. Cuscuta campestris Yuncker 


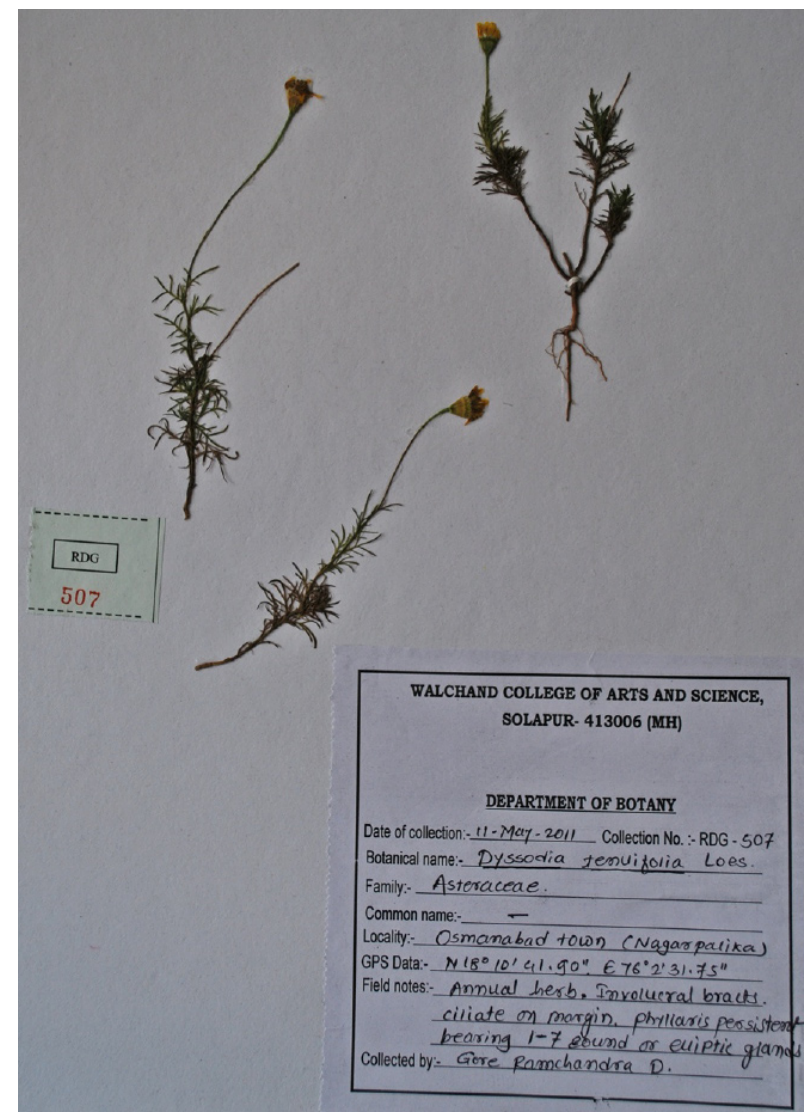

Image 11. Dyssodia tenuifolia

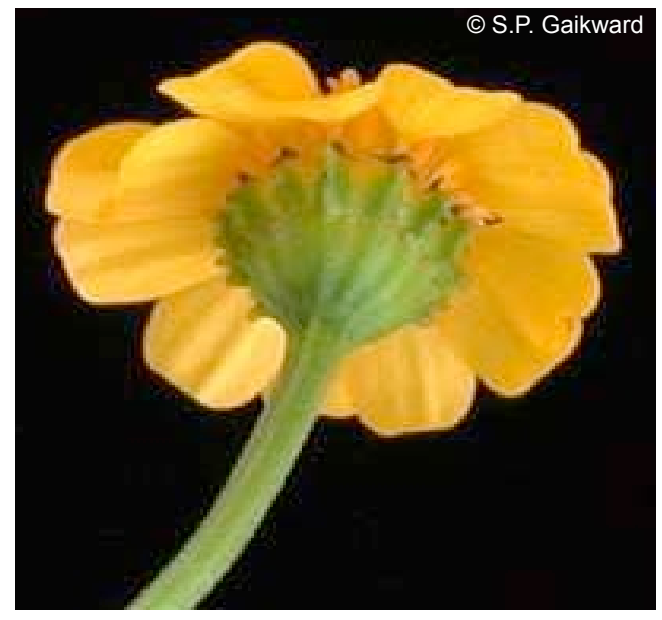

Image 12. Dyssodia tenuifolia Loes.

\section{2'31.75”'E).}

Note: Peculiar yellow oil glands are present on the outer side of involucral bracts.

Eulophia graminea Lindl. Gen. Sp. Orchid. 182. 1833. (Orchidaceae) (Images $13 \& 14$ )

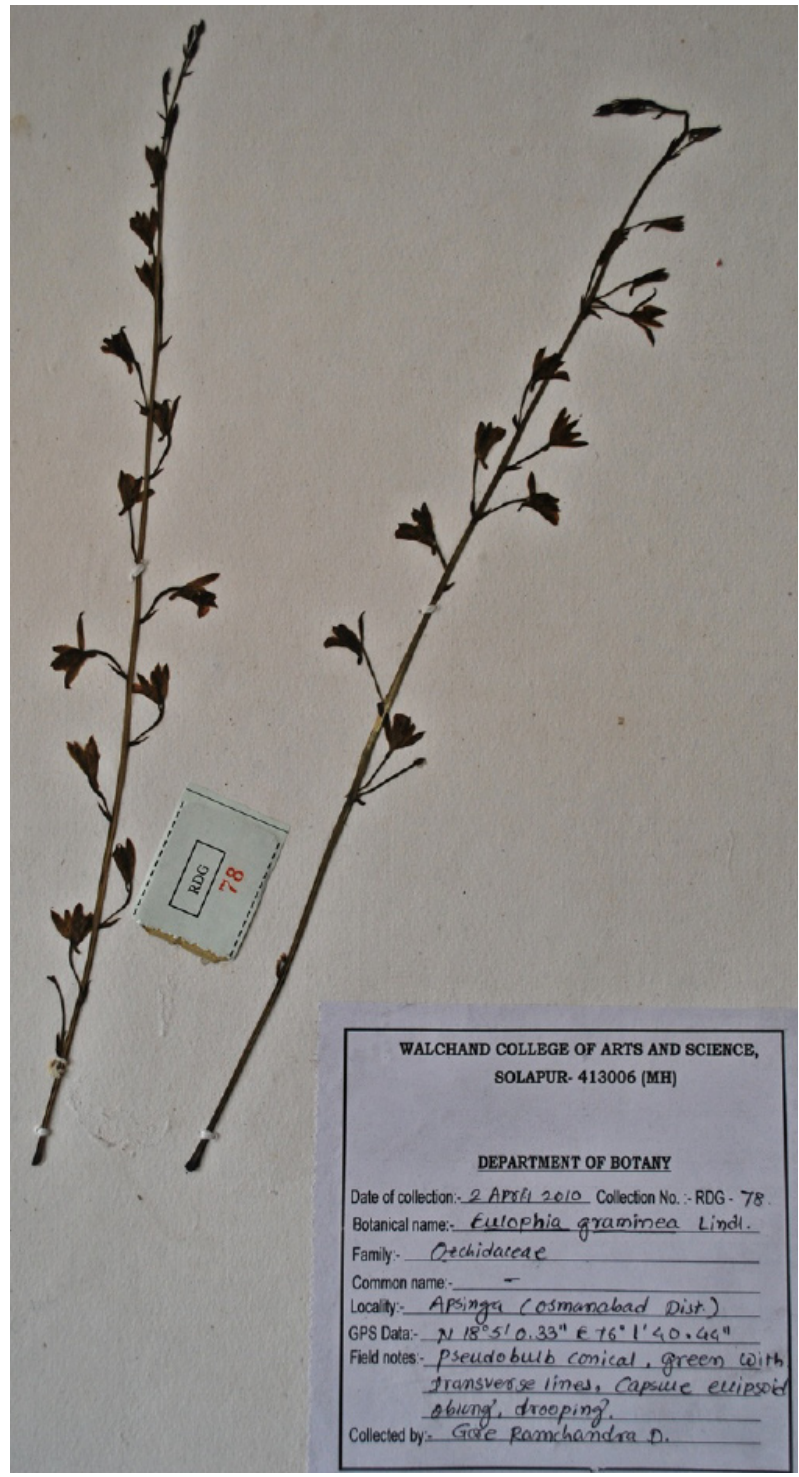

Image 13. Eulophia graminea

Pseudobulbs conical, green, marked with transverse lines of leaf bases. Leaves linear. Scapes lateral, 1-2 per pseudobulb. Capsules $2-2.5 \times 0.7-0.8 \mathrm{~cm}$, ellipsoid-oblong, drooping.

Flowering and Fruiting: September-April.

Distribution: Apsinga $\left(18^{0} 5^{\prime} 0.33^{\prime} \mathrm{N} \quad \&\right.$ 7601'40.44'E) in Osmanabad District.

Note: Bachulkar \& Yadav (1993) had reported this orchid from sugarcane fields near Islampur of Sangli District where they had seen only two individuals. The present collection confirms its occurrence as well as its extended distribution in Maharashtra. However, it was uncommon on wet margins of temporary running water streams.

Specimen examined: 02.iv.2010, RDG-78. 


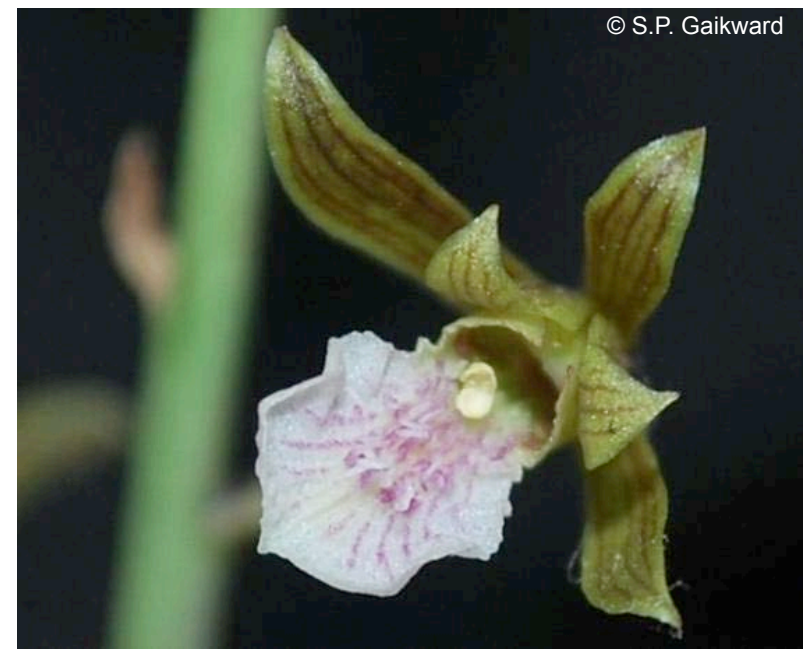

Image 14. Eulophia graminea Lindl.

Iphigenia magnifica Ansari \& Rolla in Bull. Bot. Surv. India 20: 162, t. 1. (1978) 1979. (Liliaceae)

(Images 15 \& 16)

Specimen examined: 06.xii.1983, Shindewadi Kuran near Malegaon (Nashik) Lakshminarasimhan 166228; 03.x.2010, RDG-248.

Erect, bulbous, herbs. Leaves linear-lanceolate. Flowers dark brownish-purple; filaments glabrous. Capsules ellipsoid-oblong, many seeded. Seeds subglobose, brown.

Flowering and Fruiting: September-February.

Distribution: Ramling (18'17'21.70'N \& 75056'39.26”E) in Osmanabad District.

Note: Iphigenia magnifica Ansari \& Rolla is endemic to Maharashtra and Karnataka, and regionally vulnerable (Mishra \& Singh 2001). It is distinct from I. indica (L.) A. Gray by having glabrous filaments.

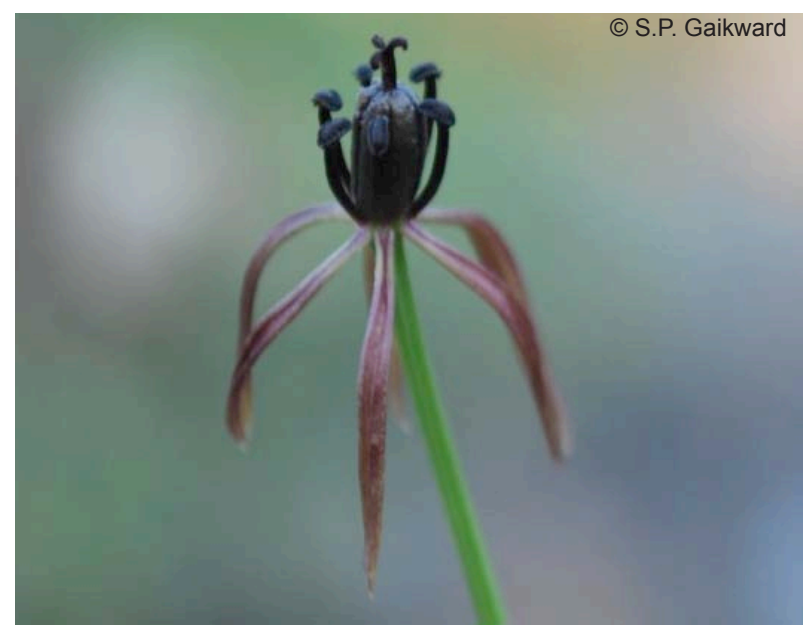

Image 15. Iphiginia magnifica Ansari \& Rolla.

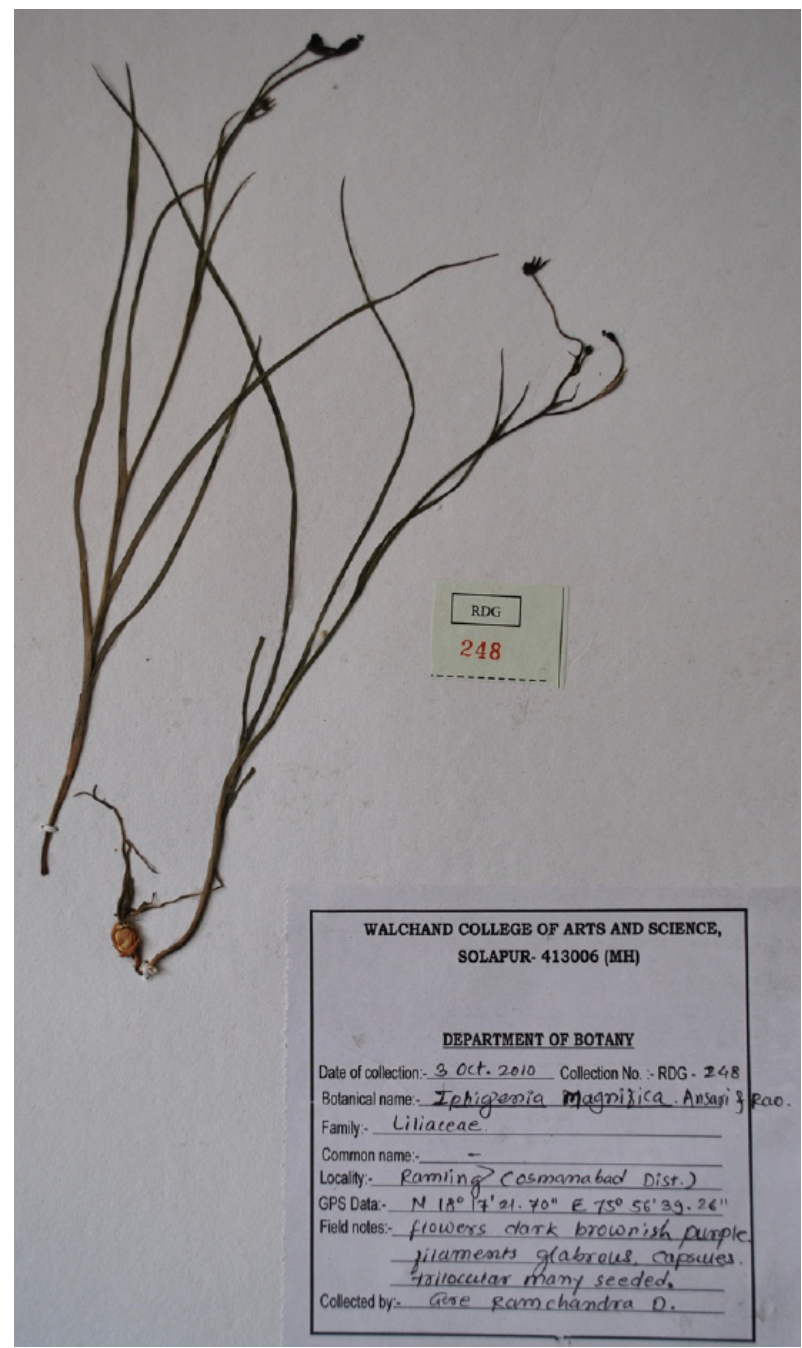

Image 16. Iphigenia magnifica

Lavandula bipinnata O. Ktze. Rev. Gen. P1. 521.

1891. var. bipinnata (Lamiaceae) (Image 17)

Specimen examined: 05.xii.2010, RDG-396.

Erect, pubescent herbs. Leaves pinnatipartite or deeply divided. Flowers bracteolate; bracts equal to or shorter than calyx, awned. Calyx tubular, hairy. Corolla Pale blue or white, 5-lobed.

Flowering and Fruiting: October-February.

Distribution: Ghatangri $\left(18^{0} 13^{\prime} 17.71^{\prime} \mathrm{N} \quad \&\right.$ 7601'15.26”E) \& Apsinga (1803'17.28”N \& $\left.76^{0} 3^{\prime} 54.30^{\prime \prime} \mathrm{E}\right)$ in Osmanabad District.

Lens culinaris Medik. Vorles. Churpf. Phys. Ocon. Ges. 2: 361. 1787. (Fabaceae) (Images 18 \& 19)

Specimen examined: 26.vi.1964, Near Vaval Dam, Pune District, B.V. Reddi 97922, (BSI Pune); 02.iii.2011, RDG- 461.

Pubescent, sub-erect herbs. Leaflets 4-6 paired; 


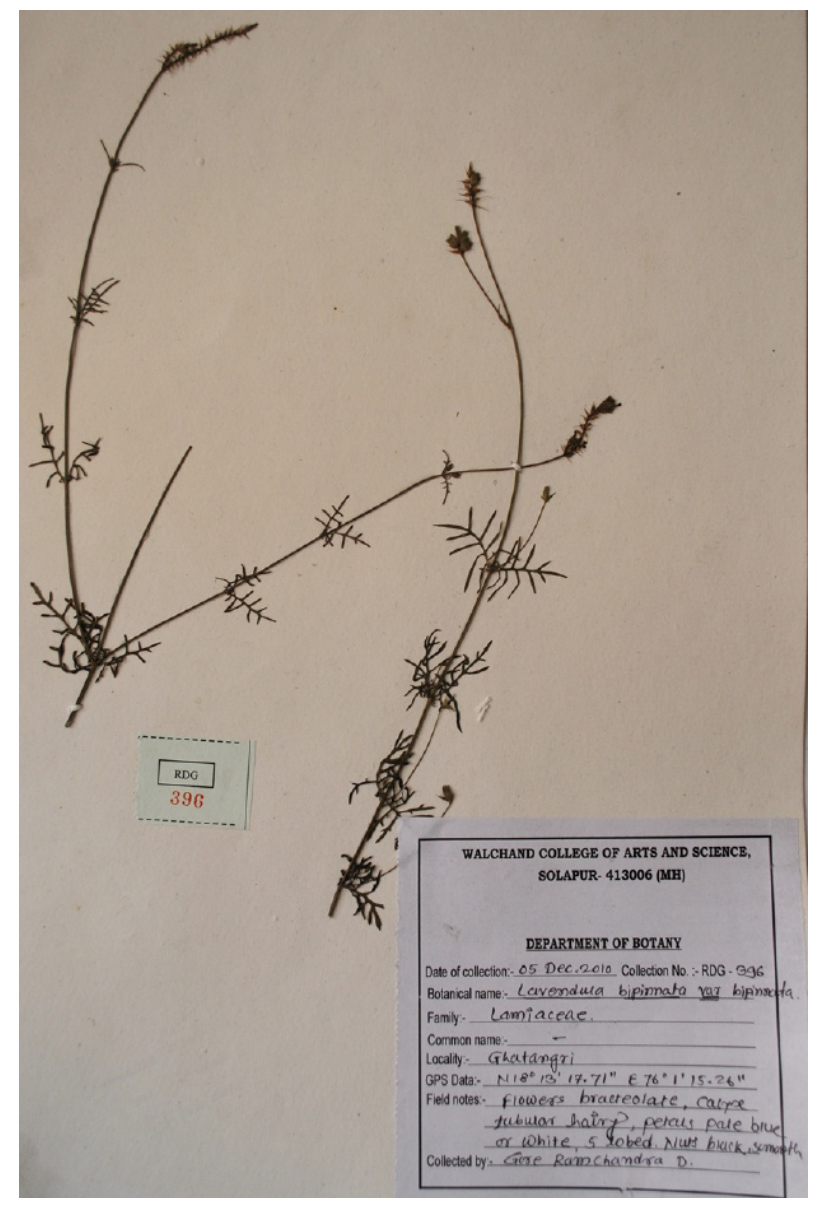

Image 17. Lavendula bipinnata var. bipinnata

rachis terminating into tendril. Raceme 1-3 flowered. Flowers white or blue-purple. Pods compressed, 2-seeded.

Flowering and Fruiting: December-March.

Distribution: Bembli (1809'11.49'N 76011'53.79'"E) in Osmanabad District.

Note: It was found occasionally in sugarcane fields.

Physalis pubescens L. Sp. Pl. 183. 1753.

(Solanaceae) (Images $20 \& 21$ )

Specimen examined: Nasik Road H. Santapau 18378-9 (Blat.); 24.x.2010, RDG-322.

Branched herbs. Leaves simple, broadly ovate, hairy, long petioled, margins serrate. Flowers solitary, axillary. Calyx lobes exceeding the berry; corolla tube rounded. Fruit a berry.

Flowering and Fruiting: August-January.

Distribution: Pohaner $\quad\left(18^{\circ} 6^{\prime} 58.32\right.$ 'N \& 76 1'6.53”E), Osmanabad (18011'12.16”N \&

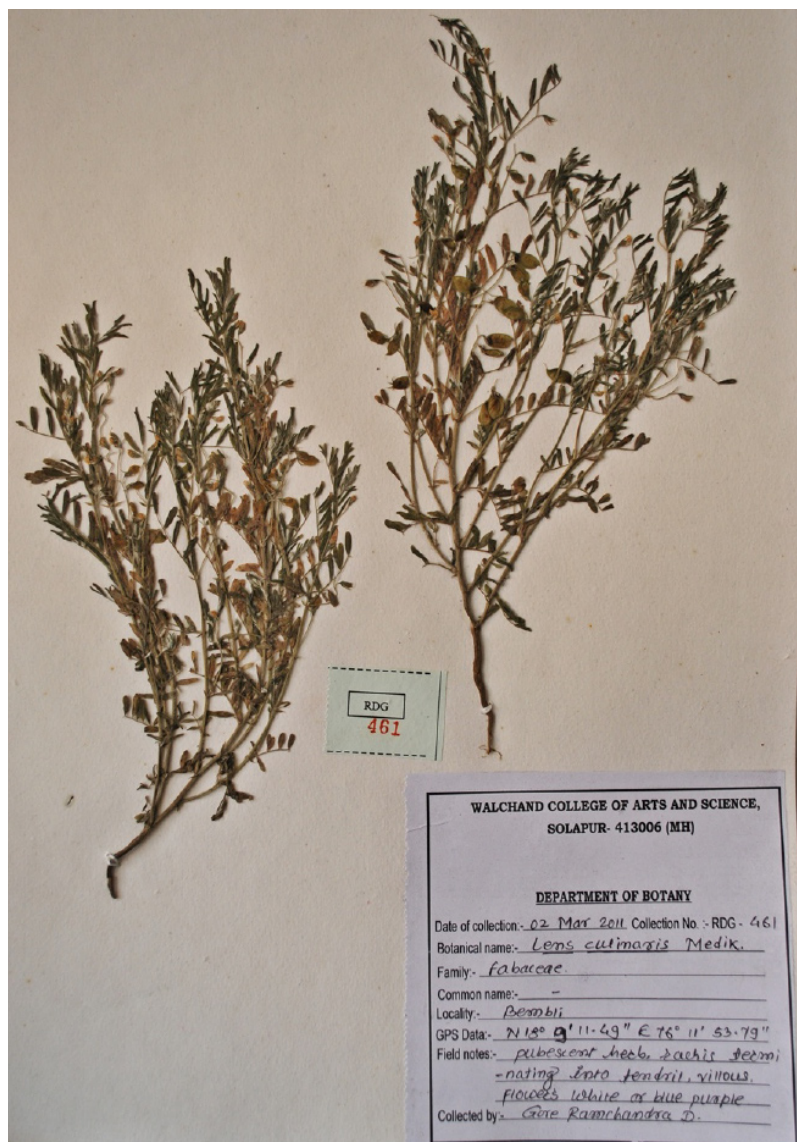

Image 18. Lens cullinaris

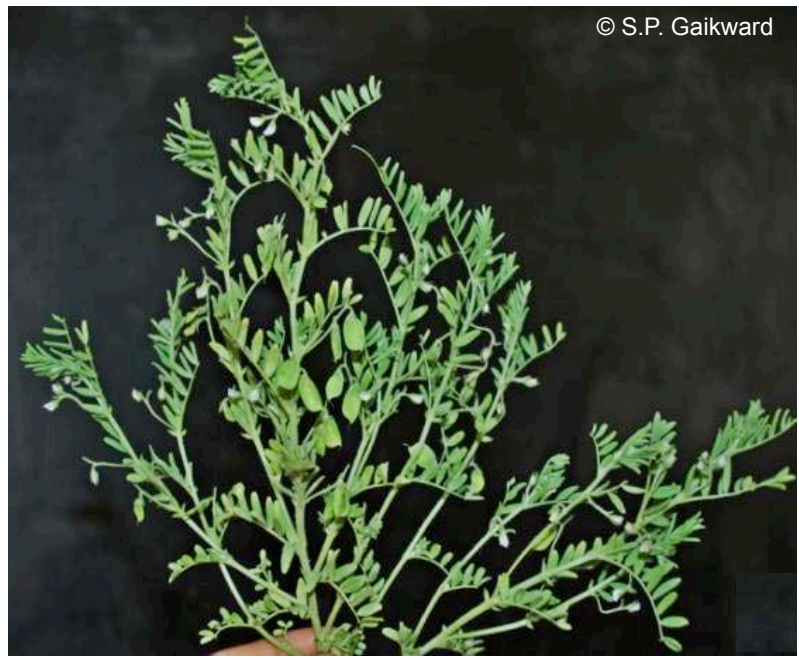

Image 19. Lens culinaris Medik.

$\left.76^{\circ} 2^{\prime} 17.52^{\prime \prime} \mathrm{E}\right)$ and Apsinga (1803'40.73"N \&

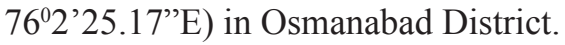

Note: It is common along road sides and in waste land. The species is distinct in having a stout stem and pubescence all over the body. 


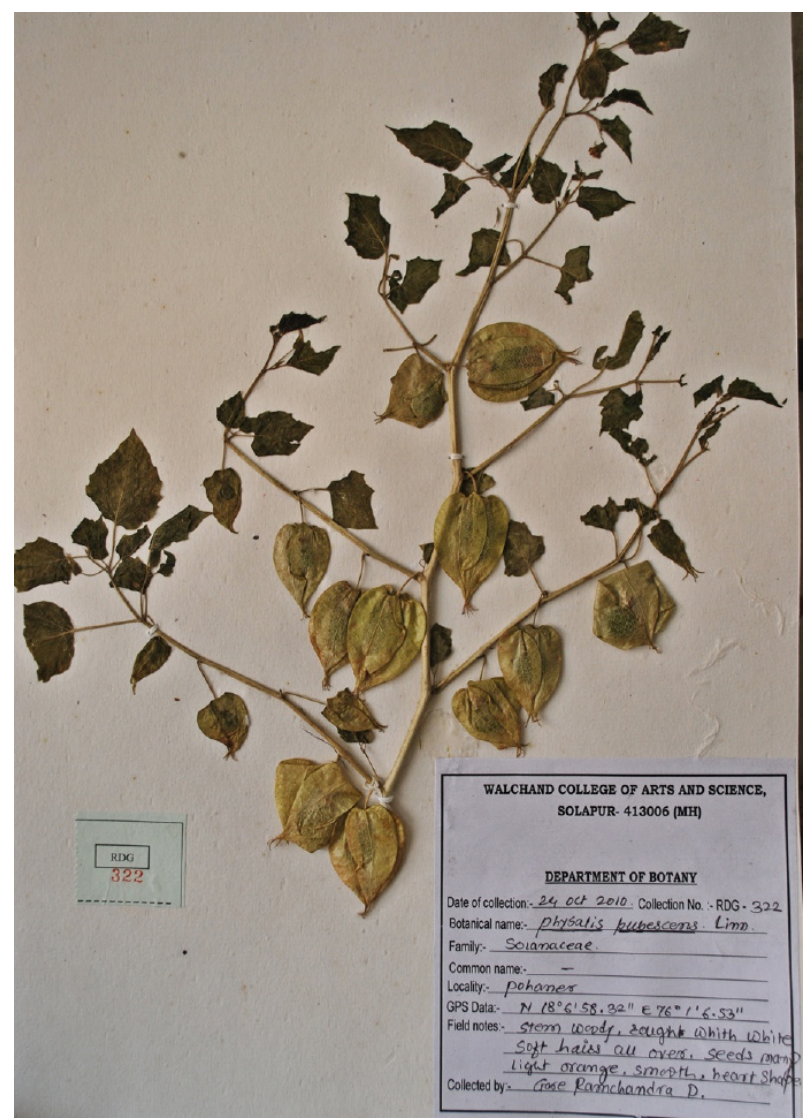

Image 20. Physalis pubescens

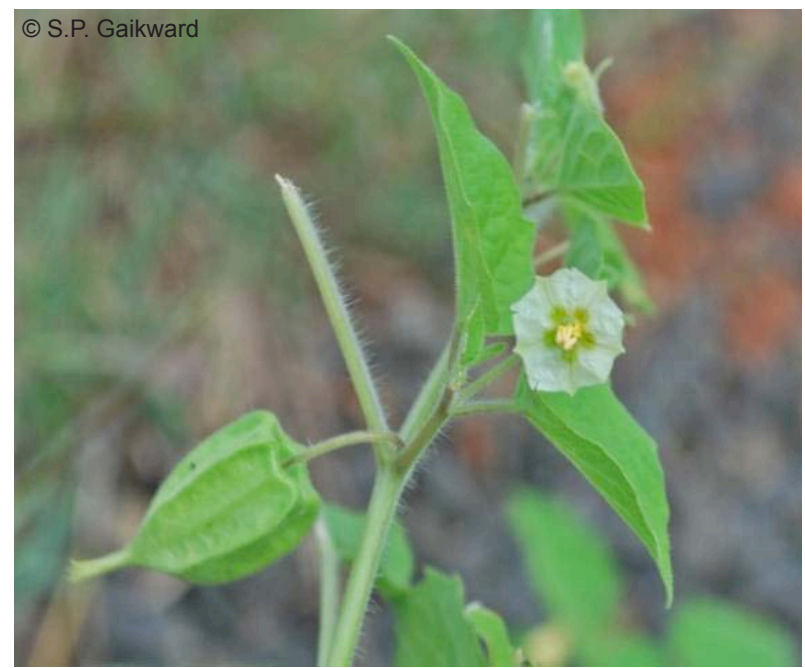

Image 21. Physalis pubescens

Synedrella vialis (Less.) A. Gray in Proc. Amer. Acad. 17: 217. 1882. Calytocarpus vialis Less. Syn.

221. 1832. (Astraceae) (Image 22)

Specimen examined: Pune (M.R. Almeida-1576, BNHS); 06.iii.2011, RDG-465.

Annual, spreading herbs, scabrid-hairy. Leaves

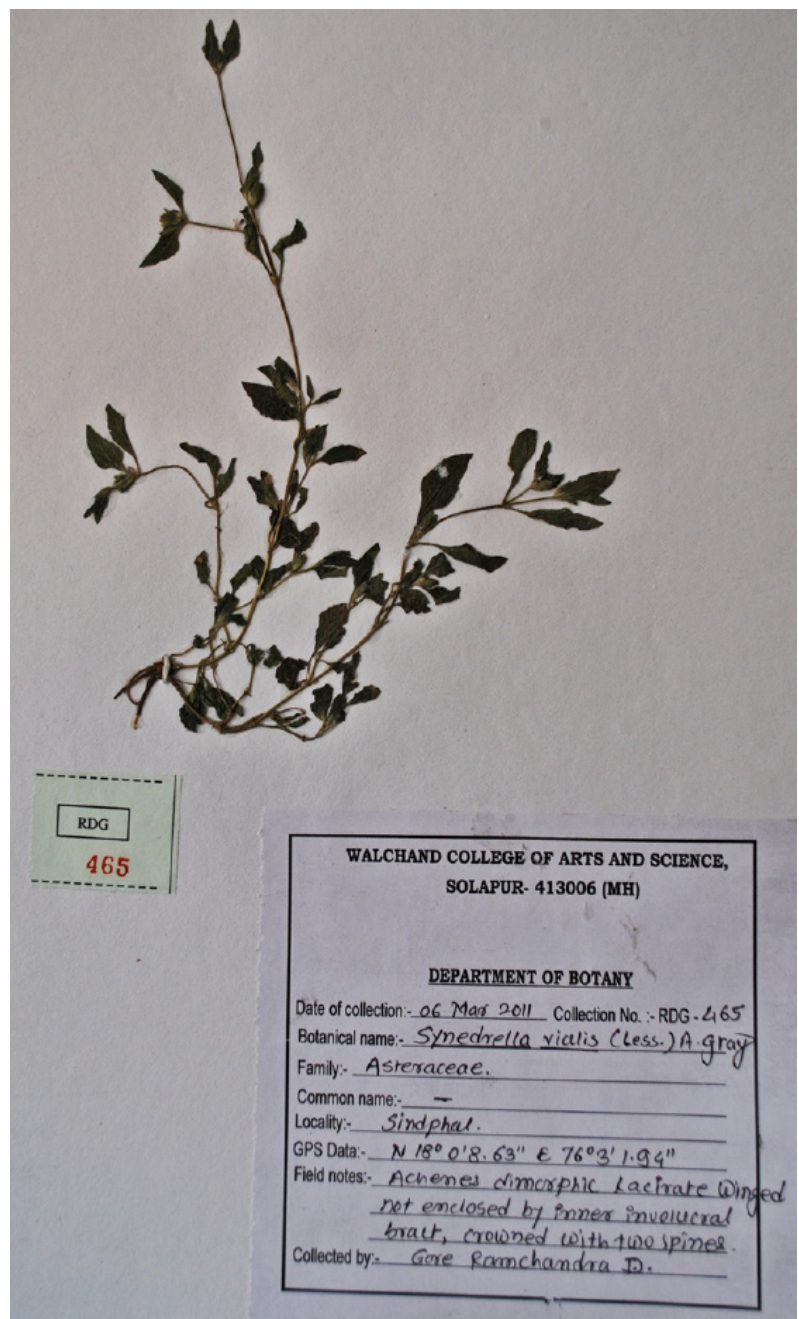

Image 22. Synedrella vialis

3-nerved from base, ovate-lanceolate. Heads axillary, solitary. Achenes dimorphic, crowned with two spines.

Flowering and Fruiting: July-September.

Distribution: Sindphal $\quad\left(18^{\circ} 0^{\prime} 8.63^{\prime \prime N} \quad\right.$ \& 76 $3^{\circ}$ '1.94”E) in Osmanabad District.

Tragia involucrata L. Sp. Pl. 980. 1753.

(Euphorbiaceae) (Images 23 \& 24)

Specimen examined: 25.ii.1959, Ameni Island, Wadhwa 49030 (BSI Pune); 02.i.2011, RDG-431.

Perennial herbs, hispid with stinging hairs; stem twining. Leaves broadly ovate, base rounded or cordate, margins serrate hairy. Flowers in hairy racemes; male in upper part and female in lower part of racemes. Capsules 3-lobed.

Flowering and Fruiting: October-January.

Distribution: Naldurg (17048'23.76”N \& 


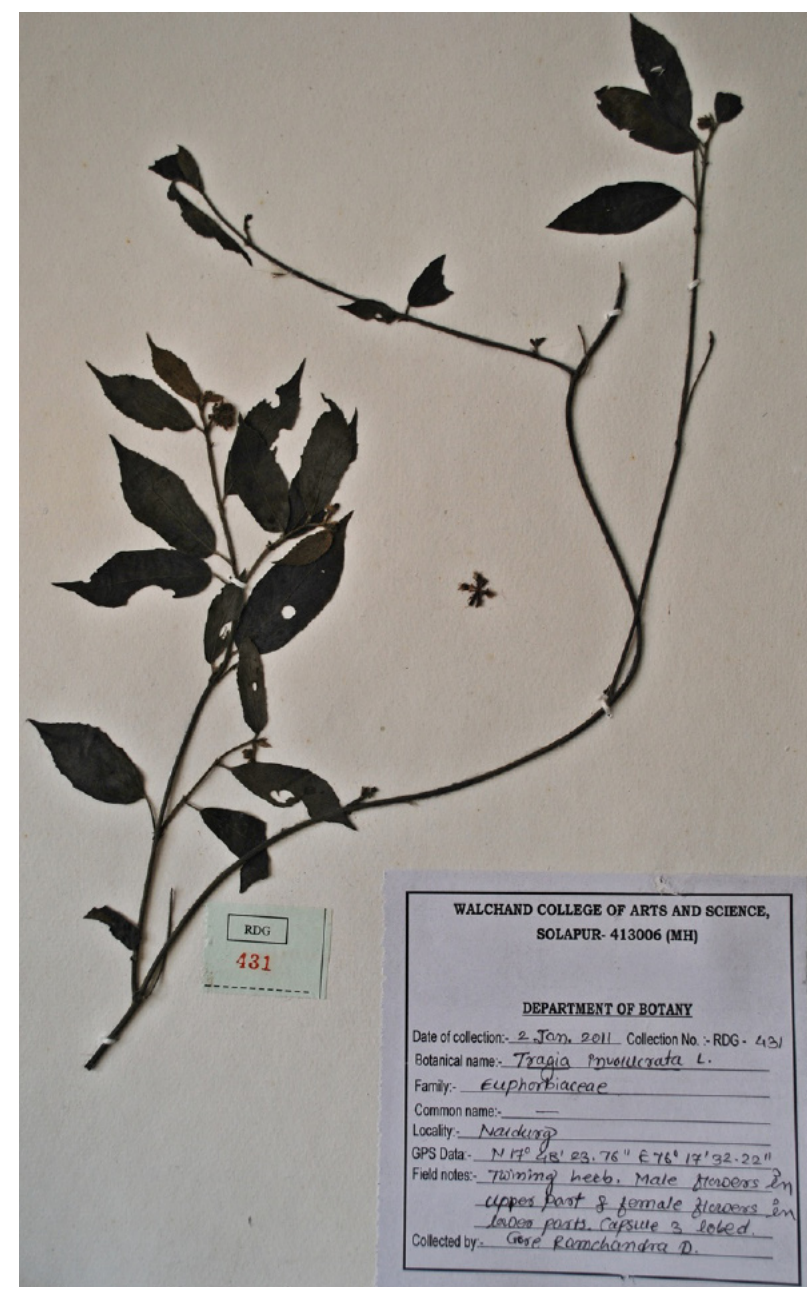

Image 23. Tragia involucrata

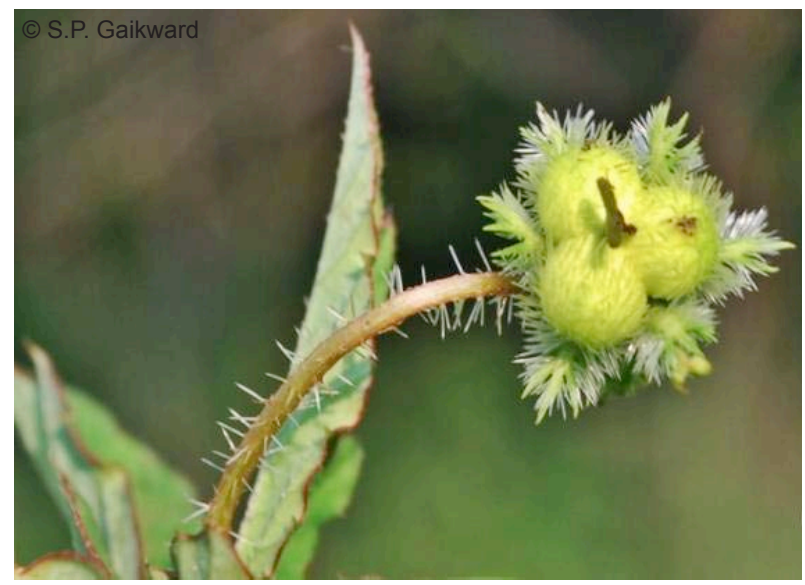

Image 24. Tragia involucrata

76017'32.22”'E) in Osmanabad District.

\section{REFERENCES}

Almeida, M.R. (1998). Flora of Maharashtra-Vol. 2. Blatter Herbarium, St. Xavier's College, Mumbai, pp. 102, 207, 208, 282.

Almeida, M.R. (2001). Flora of Maharashtra-Vol. 3 a \& b. Blatter Herbarium, St. Xavier's College, Mumbai, pp. 116, 301, 138, 371, 904.

Almeida, M.R. (2003). Flora of Maharashtra-Vol. 4a. Blatter Herbarium, St. Xavier's College, Mumbai, 196pp.

Almeida, M.R. (2009). Flora of Maharashtra-Vol. 5a. Blatter Herbarium, St. Xavier's College, Mumbai, pp. 47, 183.

Bachulkar, M.P. \& S.R. Yadav (1993). Some new plant records for Maharashtra. Journal of Economic and Taxonomic Botany 17: 329 .

Cooke, T.C. (1958a reprint edition) Flora of the Presidency of Bombay Presidency-Vol. 1. Botanical Survey of India, Kolkata, pp. 435, 474, 478.

Cooke, T.C. (1958b reprint edition) Flora of the Presidency of Bombay Presidency -Vol. 2. Botanical Survey of India, Kolkata, 577pp.

Cooke, T.C. (1958c reprint edition) Flora of the Presidency of Bombay Presidency -Vol. 3. Botanical Survey of India, Kolkata, 119pp.

Lakshminarasimhan, P. (1996). Flora of Maharashtra, Monocotyledons-Series 2. Botanical Survey of India, Kolkata, pp. 28, 139.

Mishra, D.K. \& N.P. Singh (2001). Endemic and Threatened Flowering Plants of Maharashtra. Botanical survey of India, Kolkata. 236-238pp.

Naik, V.N. (1966). A new Crotalaria species from Osmanabad District. Indian Forestry 92(12): 790-791.

Naik, V.N. (1967). Amaranthus polygonoides L. from Osmanabad district, a new record for India. Journal of the Bombay Natural History Society 64(1): 134-135.

Naik, V.N. (1969). An artificial key to the Leguminosae of Osmanabad district. Marathwada University Journal of Science 8(1): 15-19.

Naik, V.N. (1970). A census of Crotalaria species in Osmanabad district. Marathwada University Journal of Science 9: 1518.

Naik, V.N. (1979). Flora of Osmanabad. Venus publishers, Aurangabad, 464pp.

Naik, V.N. (1998). Flora of Marathwada-Vols. 1 \& 2. Amrut Prakashan, Aurangabad, 1182pp.

Singh N.P \& S. Karthikeyan (eds.) (2000). Flora of Maharashtra-Vol. I. Series 2. Botanical Survey of India, Kolkata, pp. 771, 826, 976.

Singh N. P \& S. Karthikeyan (eds.) (2001). Flora of Maharashtra-Vol. II. Series 2. Botanical Survey of India, Kolkata, pp. 31, 205, 245, 490, 581, 722, 780. 